\title{
5 years of experience with DIBH (Deep inspiration breath-hold) combined with SGRT (Surface-Guided Radiation Therapy) in left-sided breast cancer
}

\section{Jahre Erfahrung mit DIBH (Deep Inspiration Breath-Hold) kombiniert mit SGRT (Surface-Guided Radiation Therapy) bei linksseitigem Brustkrebs}

Authors

Claudia Steffal, Annemarie U. Schratter-Sehn, Karin Brinda-Raitmayr, Thomas Kann, Daniela Mailat, Jochen Reiterer, Günther Tremmel

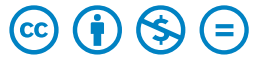

Affiliation

Institut für Radioonkologie, Sozialmedizinisches Zentrum Süd, Kaiser-Franz-Josef-Spital und Geriatriezentrum Favoriten, Vienna, Austria

Key words

breast cancer, breast irradiation, cardiotoxicity, DIBH, SGRT

Schlüsselwörter

Brustkrebs, Brustbestrahlung, Kardiotoxizität, DIBH, SGRT

\author{
Bibliography \\ DOI https://doi.org/10.1055/a-0849-0524 \\ Senologie 2020; 17: 14-22 \\ (c) Georg Thieme Verlag KG, Stuttgart · New York \\ ISSN 1611-6453
}

\section{Correspondence \\ Dr. Claudia Steffal \\ Spitalsoberärztin}

Institut für Radioonkologie

Sozialmedizinisches Zentrum Süd

Kaiser-Franz-Josef-Spital und Geriatriezentrum Favoriten,

Kundratstr. 3, A-1100 Vienna, Austria

claudia.steffal@wienkav.at

\section{ZUSAMMENFASSUNG}

Hintergrund Die Radiatio bei Patientinnen mit einem linksseitigen Mammakarzinom ist mit einer erhöhten kardialen Mortalität und Morbidität vergesellschaftet. Die DIBH-Technik (Deep Inspiration Breath-Hold) in Kombination mit SGRT (Surface-Guided Radiation Therapy, Catalyst-System) könnte deutliche dosimetrische Vorteile für Herz, LAD und die ipsilaterale Lunge gegenüber einer Bestrahlung in normaler Atmung haben. Deshalb wurde diese Technik im Oktober 2013 am Institut für Radioonkologie des KFJ/SMZ-Süd Wien eingeführt und klinisch implementiert.

Material und Methoden Von Oktober 2013 bis Dezember 2018 wurden 548 Patienten nach konservativer Operation von linksseitigem invasivem Brustkrebs zur Strahlentherapie überwiesen. Alle Patienten gaben ihr schriftliches Einverständnis und erhielten unabhängig vom Alter oder respiratorischen Erkrankungen Trainingseinheiten für die DIBH-Technik. Bei Nichtgelingen erfolgte die Planung in normaler Atemlage (NB). Verglichen wurde die relative Dosisreduktion am Herzen und der ipsiplateralen Lunge. Akute Nebenwirkungen wurden gemäß RTOG, Spätreaktionen nach CTCAE Version 4.03 ausgewertet.

Ergebnisse Das Durchschnittsalter der DIBH-Patienten betrug 58 Jahre (27-90a), der NB-Patienten 65 Jahre (30-80a). Die Nachsorge wurde bis Juni 2019 durchgeführt. Das mediane Follow-up betrug 52 Monate (Range 7-73 m). Ausgewertet wurden die mittleren Dosen (Dmean) für die Risikoorgane linke Lunge und Herz. Die Werte des Dmean an der linken Lunge lagen bei DIBH-Technik im Median bei 6,91 Gy (Range 1,44-12,4 Gy), am Herzen im Median bei 1,17 Gy $(0,12-$ 3,19 Gy). Bei den NB (normal breathing, free breathing)-Plänen waren die Dmean-Werte der ipsilateralen Lunge bei 8,92 Gy $(5,23-16,9$ Gy), der Dmean lag am Herzen bei 2,31 Gy (0,71-4,21 Gy), was einer Reduktion um die Hälfte entspricht. Die Akutreaktionen waren vergleichbar: RTOG 1: $70,8 \%$ vs. $64 \%$, RTOG $36,6 \%$ vs. $5,6 \%$, no reaction $3,2 \%$ vs. $1,4 \%$. Bei den CTCAE-1-NW gab es deutlich mehr Spätreaktionen in der NB-Gruppe (51,6\% vs. 12,67\%).

Schlussfolgerung Die Deep Inspiration Breath-hold (DIBH)Technik mit Surface-Guided Radiation Therapy (SGRT) ist eine einfache, reproduzierbare Methode mit hoher Akzeptanz bei den Patienten. Die mittlere Dosisbelastung am Herzen und an der linken Lunge kann damit deutlich reduziert werden, am Herzen sogar um die Hälfte.

\section{ABSTRACT}

Background Radiation treatment to the left breast is associated with increased cardiac morbidity as well as mortality. Deep inspiration breath-hold (DIBH) technique with Surface Guided Radiation Therapy (SGRT) could have dosimetric advantages over the free breathing technique (NB, normal breathing) in cardiac (heart and LAD) and ipsilateral lung sparing in patients 
with left-sided breast cancer after surgery. Therefore this technique was implemented in 2013 at the institute of radiooncology at the KFJ/SMZ-South - Hospital Vienna.

Methods From Oct 2013 - December 2018548 patients were referred to radiotherapy following conservative operation of left-sided invasive breast cancer. All patients gave their informed consent and underwent training sessions for the DIBH-technique independent of age or breathing activity or respiratory disorders. Patients who turned out to be unfit for DIBH were enrolled for NB. The relative reduction in Dmean heart and left lung dose was compared between the two cohorts. Acute radiation induced side effects were classified according to the Radiation Therapy Oncology Group/The European Organisation for Research (RTOG) [37]; late toxicity rates according to the Common Terminology Criteria for Adverse Events (CTCAE Version 4.03)

Results The median age of the DIBH-patients was 58 years (27-90), of the NB-patients $65(30-80)$ years. Follow-up was obtained until June 2019. The median follow-up was 52 months (range $7-73 \mathrm{~m}$ ). The average coverage of Dmean left lung was $6.91 \mathrm{~Gy}$ (1.44 Gy - 12.4Gy). The average coverage of Dmean heart was $1.17 \mathrm{~Gy}(0.12 \mathrm{~Gy}-3.19 \mathrm{~Gy})$ in the DIBH-cohort. The NB - plans had a Dmean of 8.92 Gy (5.23-16.9 Gy) at the ipsilateral lung and a Dmean of 2.31 Gy (0.71-4.21 Gy) at the heart. This shows that the DIBH-technique halved the Dmean of the heart. The amount of acute side effects was comparable between the two groups: RTOG 1: $70.8 \%$ vs. $64 \%$, RTOG 3 $6.6 \%$ vs. $5.6 \%$, no reaction $3.2 \%$ vs. $1.4 \%$. There were more CTCAE 1-late events in the NB-group ( $51.6 \%$ vs. $12.67 \%$ ).

Conclusion Deep inspiration breath-hold (DIBH) technique with Surface Guided Radiation Therapy (SGRT) is a rather simple, reproducable method with a high acceptance of the patients who can actively participate in the whole treatment process. The mean dose at the heart and the left lung can be reduced, at the heart even by as much as $50 \%$.

\section{Background}

Worldwide breast cancer is the most common cancer affecting women. Adjuvant radiotherapy plays a central role after breast conserving surgery in early-stage breast cancer. A considerable number of trials [12, 13, 18, 19, 23, 24, 29, 34] has shown on the one hand a reduction in recurrence and death rates from breast cancer and on the other hand an improvement in overall survival with adjuvant radiotherapy. A large meta-analysis by the Early Breast Cancer Trialist's Group [23] found that patients treated with radiation therapy after breast-conserving surgery (BCS) had a $7 \%$ chance of local recurrence after 5 years compared with $26 \%$ in patients who did not undergo radiotherapy. Additionally they had an absolute risk reduction of $5.4 \%$ in breast related mortality with radiation therapy after BCS compared with BCS alone.

Despite its improvements, radiotherapy to the breast can cause side effects. A long-term complication of left breast irradiation is the risk of cardiac mortality and morbidity as well as pulmonary complications as multiple epidemiological studies have shown $[18,24]$. Darby et al. [16] found after retrospectively analyzing 2168 patients from the Nordic cancer registry that the relative risk for ischemic heart disease increased by $7.4 \%$ for every 1 Gray (Gy) in mean heart dose. However especially the Ramus interventricularis anterior (RIVA; left anterior descending coronary artery- LADCA) has a high risk of obtaining significant higher doses and is responsible for the development of pathologic blood vessel disorder and myocardial or coronary artery disease [1, 20-22].

Modern radiation treatment techniques may reduce the dose to the heart. For this purpose the deep inspiration breath-hold technique has made considerable efforts to reach this goal in minimizing the dose to the heart $[3-11,14]$. The technique is based upon the observation that during inspiration, the flattening of the diaphragm and the expansion of the lungs pull the heart away from the chest wall. Deep inspiration breath hold (DIBH) is a radiation therapy technique where patients take a deep breath during treatment to a specific threshold and hold this level of inspiration during every radiation therapy field delivered. By taking a deep breath in, the lungs fill with air and thus the heart will move away from the chest.

SGRT (Surface Guided Radiation Therapy) is a system to position and monitor the patient's external surface during their radiation treatments to ensure they are in the correct position. It matches surface data in real-time and tracks the patient in all six degrees of freedom to a reference model captured either during the planning process or using the internal imaging in the treatment room. SGRT can be used both in setup and therapy for breast cancer patients, DIBH, SRS, SBRT, thoracic lesions and many more. With SGRT, the radiation treatment can be delivered much more accurately to the intended target for every patient than with other techniques.

The DIBH technique with SGRT (Catalyst System) was adopted and clinically implemented at the institute of radiation oncology at the KFJ/SMZ-South Vienna in October 2013 for all patients with left-sided breast-cancer. The aim of this report is to provide our experiences regarding the implementation, utilization, patient compliance, planning methods, treatment verification, dosage and limits of the DIBH-technique with SGRT. The primary goal was to evaluate if there would be a relative reduction in Dmean heart and left lung dose between the two cohorts (DIBH vs. NB).

\section{Patients and methods}

\section{Patients}

Between Oct 2013 -and December 2018548 consecutive patients with histologically confirmed breast cancer were enrolled. The patients were referred to radiotherapy following conservative surgery of left-sided invasive breast cancer. All eligible patients were informed about the potential benefit of an additional deep inspiration breath hold. Part of our daily clinical routine is that all 
patients are offered the oppurtunity to try the training session for the DIBH-technique independent of age or breathing activity or respiratory disorders. Patients who turned out to be unfit for DIBH were enrolled for NB.

Data was collected by analysing patients records. All treatments described in the present report were carried out in accordance with national law. Before starting the entire treatment procedures, all patients had to give their informed consent for the treatment in $\mathrm{DIBH}$ (deep inspiration breath hold) and also for the NB (normal breathing)-method.

\section{Treatment prescriptions}

Postoperative RT is strongly recommended after BCS [31, 32, 34]. A whole breast radiation therapy (WBRT) alone reduces the 10 -year risk of any first recurrence (including locoregional and distant) by $15 \%$ and the 15 -year risk of breast cancer-related mortality by $4 \%$ [34]. Boost irradiation gives a further $50 \%$ RR reduction. It is indicated for patients who have unfavourable risk factors for local control such as: age < 50 years, grade 3 tumours, extensive DCIS, vascular invasion or non-radical tumour excision [31, 32, 34]. Doses used for local and/or regional adjuvant irradiation have traditionally been 45-50 Gy in 25-28 fractions of 1.82.0 Gy with a typical boost dose of 10-16 Gy in 2 Gy single doses. Shorter fractionation schemes (e.g. 15-16 fractions with 2.5$2.67 \mathrm{~Gy}$ ) were also used according to the Start B protocol [17, 29, 35,36 ] or the Whelan regimen [19]. An irradiation of the axillary lymph nodes with $50 \mathrm{~Gy}$ in 25 fractions as specified by guidelines [12] was performed when indicated. Nevertheless at our institute it is extremely seldom that an irradiation of the internal mammary nodes [13] is carried out according to the AGO-and/or S3-guidelines [31, 32] - however, in this cohort no treatment of the lymph nodes of the A. mammaria interna was performed.

\section{Workflow and system}

Primarily all patients underwent the same workflow:

- Training session for DIBH: upper and lower thresholds of breathing can be fixed, positioning of respiratory marker block (with integrated infrared reflectors), audio- and video feedback

- CT-Scan in DIBH and or additionally in NB; for immobilization we used breast boards with both arms positioned above the head in a spine position

- Planning and verification (either in DIBH or if not possible in NB)

- Daily treatment

- Daily imaging with an adaptive planning approach

- Psychological support and information once a week in order to enable a return to normal life after breast cancer

At our institute the Varian Real-time Position Management ${ }^{\mathrm{TM}}$ (RPM) system and the Catalyst ${ }^{\mathrm{TM}}$ software and system from C-Rad are used. RPM is a non-invasive, video-based system that allows for clean imaging and treatment of lung, breast, and upper abdominal sites. The RPM system accommodates both breath hold and free breathing protocols. The Catalyst system for real time patient tracking captures the complete body surface of the patient continuously, compares the current patient posture and position to the previously recorded reference setup in real-time,

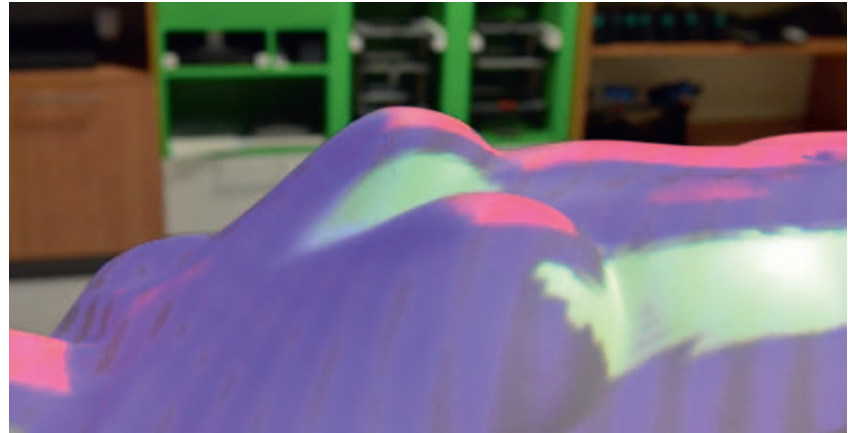

- Fig. 1 SGRT using a phantom for real time patient tracking at KFJ Vienna; the tumour position can be correlated in relation to the patient's respiratory cycle.

detects intra-fraction motion and is also used for respiratory gating as shown in $\mathbf{F i g}$. 1. The unit is mounted on the ceiling where it has an unobstructed view of the patient independent of any motion of the gantry or its on-board imagers. The Catalyst ${ }^{\mathrm{TM}}$ software has a tight connection and integration with the Varian RPM system. The specially designed filter monitors can predict the patient's breathing pattern and can account for the patient's coughing or changes from the predicted breathing pattern. The tumour position can be correlated in relation to the patient's respiratory cycle. Using an infrared tracking camera and a reflective marker, the system measures the patient's respiratory pattern and range of motion and displays them as a waveform.

The gating thresholds are set when the tumor is in the desired position of the respiratory cycle. These thresholds determine when the gating system turns the treatment beam on and off [25-27] as illustrated in > Fig. 2.

In this way the dose to the heart can be minimized in our breast treatments, the maximum heart distance of the heart irradiated is less than $1 \mathrm{~cm}$ ( $>$ Fig. 3 ).

Furthermore the patients are treated with two identical Varian Truebeam linear accelerators with an integrated "Perfect Pitch" (6-DoF-couch), a computer-controlled robotic treatment couchtop with remote positioning correction and 6 degrees of freedom [28]. The modern planning techniques included IMRT (intensity modulated radiotherapy)-, FIF (field in field) - and standard techniques. The targets (whole breast or chest wall + /- axillary lymph nodes), organs at risk (ipsilateral lung, contralateral lung, heart and contralateral breast) and other organs of interest were delineated as per the RTOG (Radiation Therapy Oncology Group) contouring recommendations [33].

\section{Follow up schedule and statistical analysis}

Our routine care programme lasts on average ten years; follow up was routinely scheduled every 3 months in the first year following radiotherapy, every 6 months in the second year and at 12-month intervals thereafter. If possible, acute radiation induced side effects were classified according to the Radiation Therapy Oncology Group/The European Organisation for Research (RTOG) [37]; late toxicity rates according to the Common Terminology Criteria for Adverse Events (CTCAE Version 4.03) [38]. Toxicity was considered early if it occurred within the first 90 days from the start of 


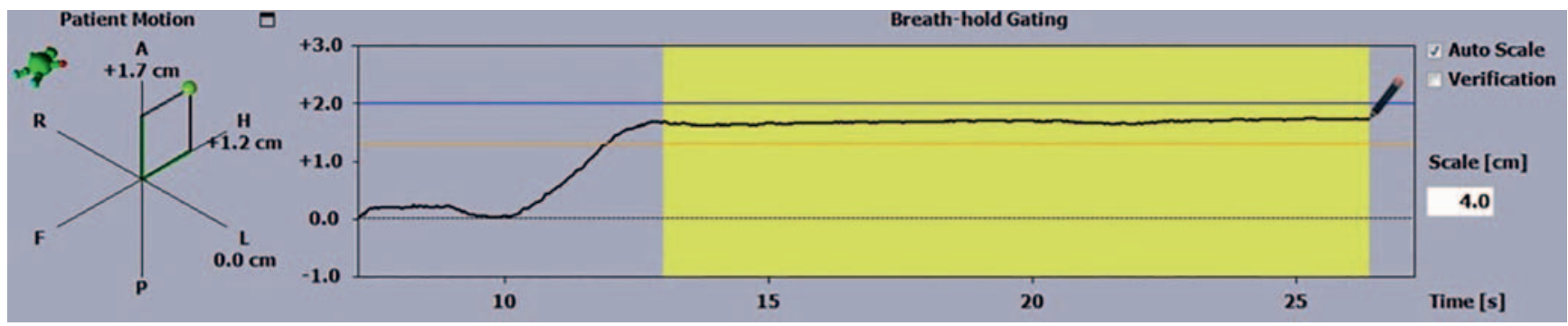

Fig. 2 Respiratory pattern in DIBH (deep-inspiration-breath-hold)-technique - deep inspiration within upper and lower thresholds; "beam" on is illustrated by yellow bar.

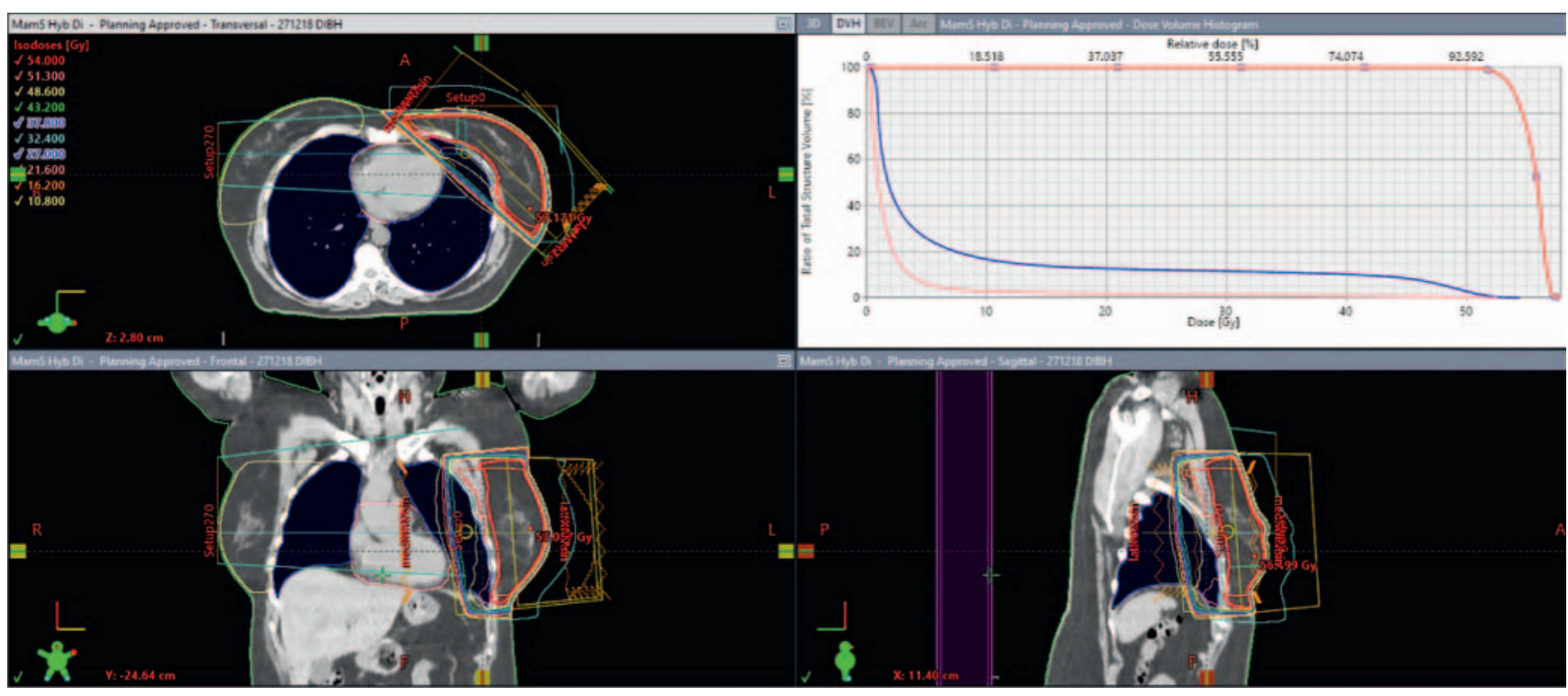

- Fig. 3 Radiotherapy of left-sided breast cancer in DIBH - maximum heart distance of heart irradiated is less than $1 \mathrm{~cm}$.

radiotherapy. Other assessments include: the complete new personal medical history, a physical examination, imaging, a full blood count, liver and renal function tests, alkaline phosphatase and calcium levels, an assessment of the menopausal status of the patient, a bone densitometry every two years and an evaluation of the cardiac function with a cardiac ultrasound or a multigated acquisition scan.

The outcomes evaluated in this retrospective analysis were primarily the feasibility and the workflow of DIBH with SGRT and secondarily, demographics as treatment-regimens and dosimetric outcomes like Dmean of the lungs and the heart. The statistical analysis was performed with the SPSS (Version 20) and Excel Office 2017; the primary goal of this analysis was an inter-cohort comparison.

The project has been displayed to the ethics committee and meets the requirements of the local guideline.

\section{Results}

\section{Demographics}

From Oct 2013 - Mai 2018548 patients were enrolled, 517 of them could receive the DIBH-technique, 31 were treated in NB.
We also treated 8 patients with right-sided breast cancer [2], 15 patients had both-sided breast cancer. Two male patients were included $(0.4 \%)$.

The median age of the DIBH-patients was 58 years (27-90), of the NB-patients 65 (30-80) years.

The majority of the patients were postmenopausal. No difference between the two groups was found regarding tumor site, TNMstaging, grading and hormonal receptor status. 100 patients received neoadjuvant systemic treatment (NACHT-regimen), 27 adjuvant chemotherapy, 453 antihormonal treatment and 50 patients had to undergo a Trastuzumab-regimen.

Almost all patients (94.3\%) had a breast-conserving surgery, $4.6 \%$ of the patients had oncoplastic techniques such as glandular adaptation, local or distant flaps or mammoplasty permitting breast remodeling. Extremely radical and radical surgical treatment of breast cancer were not investigated in this cohort. Nevertheless 30 patients ( $5.5 \%$ ) had an Ablatio mammae. Sentinel Node Biopsy as a standard procedure was performed in $98 \%$ of the cases, 18 patients received an additional Level I and/or Level II axilla dissection ( $23 \% \mathrm{~N}+$ of the patients).

The majority of the treated patients had the staging T1 (373) and N0 (416) as shown in > Table 1. There was an equal distribu- 
- Table 1 Patient, disease and treatment characteristics.

\begin{tabular}{|c|c|c|c|}
\hline & & $\begin{array}{l}\text { NB } \\
(n=31)\end{array}$ & $\begin{array}{l}\text { DIBH } \\
(n=517)\end{array}$ \\
\hline \multirow[t]{3}{*}{ Age } & Mean & 65 & 58 \\
\hline & Min & 30 & 27 \\
\hline & Max & 80 & 90 \\
\hline \multirow[t]{2}{*}{ Sex } & Male & 0 & 2 \\
\hline & Female & 31 & 515 \\
\hline \multirow[t]{5}{*}{$\mathrm{OP}$} & $A D$ & 7 & 11 \\
\hline & Ablatio & 3 & 27 \\
\hline & Sentinel & 31 & 506 \\
\hline & BET & 28 & 490 \\
\hline & Remodeling & 2 & \\
\hline Both sided & & 3 & 12 \\
\hline \multirow[t]{7}{*}{ T } & $\begin{array}{l}\mathrm{T} 1 \text { (pT1 / pT1mi/ } \\
\text { урт1) }\end{array}$ & 24 & 349 \\
\hline & T2 (рT2/ ypT2) & 1 & 76 \\
\hline & T3 (рT3 / урТ3) & 2 & 8 \\
\hline & T4 (рT4 / ypT4) & 0 & 3 \\
\hline & Tis (pTis/ypTis) & 1 & 47 \\
\hline & урТ0 & 2 & 30 \\
\hline & ypTx & 1 & 4 \\
\hline \multirow[t]{6}{*}{$\mathrm{N}$} & pNO / ypNO & 18 & 398 \\
\hline & N1 (N1 / N1a/N1mi) & 9 & 79 \\
\hline & N2 (N2a) & 1 & 2 \\
\hline & N3 (N3a) & 1 & 4 \\
\hline & $\mathrm{Nx}$ & 1 & 4 \\
\hline & No data & 1 & 30 \\
\hline \multirow[t]{4}{*}{ Grading } & G1 & 4 & 77 \\
\hline & $\mathrm{G} 2$ & 20 & 271 \\
\hline & G3 & 4 & 123 \\
\hline & No data & 3 & 46 \\
\hline \multirow[t]{3}{*}{ Herceptin } & Yes & 3 & 47 \\
\hline & No & 22 & 458 \\
\hline & No data & 6 & 12 \\
\hline \multirow[t]{3}{*}{ HRT } & Yes & 26 & 5 \\
\hline & No & 5 & 458 \\
\hline & No data & 0 & 17 \\
\hline \multirow[t]{3}{*}{ CHT } & Yes & 5 & 99 \\
\hline & No & 26 & 402 \\
\hline & No data & 0 & 16 \\
\hline \multirow[t]{4}{*}{ RTOG } & RTOG1 & 22 & 331 \\
\hline & RTOG2 & 6 & 150 \\
\hline & RTOG3 & 2 & 29 \\
\hline & No reaction & 1 & 7 \\
\hline
\end{tabular}

- Table 1 (Continuation)

\begin{tabular}{|l|l|l|l|}
\hline & $\begin{array}{l}\text { NB } \\
(\mathbf{n = 3 1 )}\end{array}$ & $\begin{array}{l}\text { DIBH } \\
(\mathbf{n = 5 1 7 )}\end{array}$ \\
\hline CTCAE & CTCAE1 & 16 & 65 \\
\hline & CTCAE2 & 1 & 38 \\
\hline & G3-5 & 0 & 0 \\
\hline & No reaction & 14 & 414 \\
\hline
\end{tabular}

HRT = Hormontherapie; $\mathrm{CHT}=$ Chemotherapie; $\mathrm{RTOG}$ = Radiation Therapy Oncology Group/The European Organisation for Research; CTCAE $=$ Common Terminology Criteria for Adverse Events.

\section{DOSAGE (GY)}

- Total dose 40.05 Gy $\quad$ Total dose 42.5 Gy $\quad$ Total dose 50 Gy

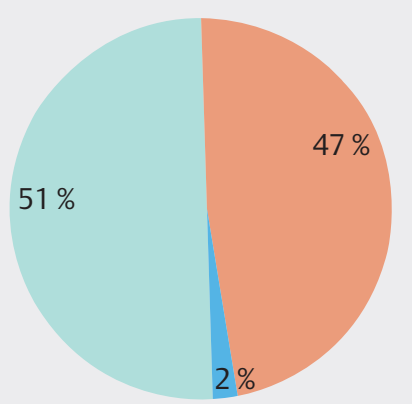

Dig. 4 Coverage of dose using 3 different RT-schemes.

tion between grading G1, G2 and G3. 40 patients suffered from heart diseases, especially coronary heart disease; 24 patients had preliminary lung problems including bronchial asthma and severe COPD. A detailed comparison of the patient-related factors of both treatment groups may be found in $>$ Table 1 .

Dose and fractionation prescribed in this analysis represent standard treatment prescriptions at our institution and the radiotherapy treatment characteristics are shown in > Fig. 4.

$51 \%$ of all 548 enrolled patients received a total dose of 50 Gy in 25 fractions + $/$ - boost irradiation (Standard protocol), $47 \%$ of 40.05 Gy in 15 fractions (Start B protocol - 17, 29, 35, 36) and $2 \%$ of 42.5 Gy in 16 fractions (Whelan protocol - 19). In 12 cases we irradiated the axillary lymph nodes with 50 Gy in 25 fractions as specified by guidelines [12]. Nevertheless it is interesting to note that $82 \%$ of the NB - patients underwent a hypofractionation protocol ( $\triangleright$ Fig. 4 ).

\section{Dosimetric outcomes}

The comparison of average dose parameters to the heart and left lung using DIBH vs. NB is summarized in > Fig. 5. Actually great efforts were taken to optimize the treatment planning on the computed tomography data sets of the patients; in order to be able to pick the best plan we decided to create at least six plans 


\section{Dmean (Gy) Heart/Lung NB}

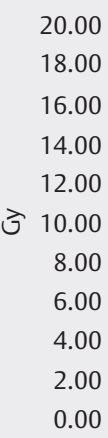

)

1.00

4.00

2.00

10.00

6.00

4.00

2.00

0.00

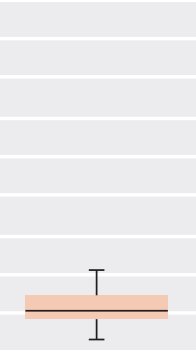

Heart Dmean (Gy)

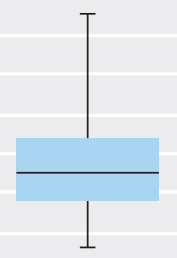

Lung Dmean (Gy)

\section{Dmean (Gy) Heart/Lung DIBH}
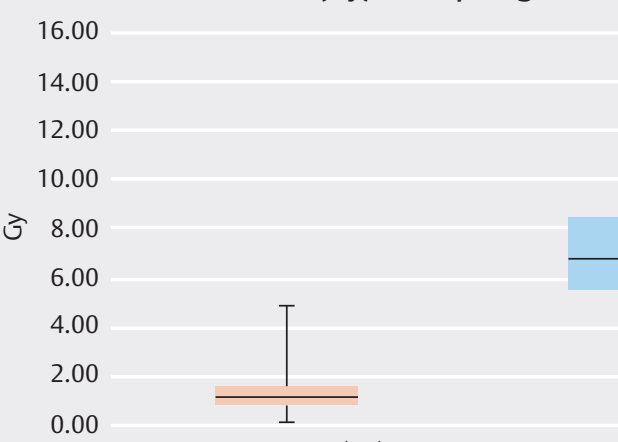

00

00

00

4.00

2.00

0.00

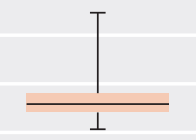

Heart Dmean (Gy)

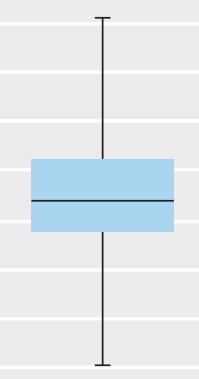

Lung Dmean (Gy)

- Fig. 5 Dmean for heart and left lung (Comparison NB vs. DIBH) - Dmean reduction heart by $45.4 \%$, ipsilateral lung by $22.5 \%$.

per patient (Range 4-12 plans), some of them in free-breathing (NB). The average coverage of Dmean left lung was 6.91 Gy (Range $1.44 \mathrm{~Gy}-12.4 \mathrm{~Gy}$ ) in the DIBH cohort. $75 \%$ of all patients were able to meet a Dmean under $8.41 \mathrm{~Gy}$. We also evaluated the NB plans. In this evaluation the Dmean left lung was $8.92 \mathrm{~Gy}$ (Range 5.23-16.9 Gy). This shows that the DIBH-technique reduced the Dmean at the ipsilateral lung around 2 Gray.

The high maximum in the DIBH group had one patient suffering from left heart failure with an enormous breast (V 2032.91 cc) and positive lymph nodes (5/12) receiving RT to the whole breast plus nodal RT. For this patient 12 different treatment plans were generated, three of them were normal breathing plans (NB) in order to be able to make a comparison it with the DIBH-plans. The Dmean left lung was much higher in the NB plans (the best NBplan had a Dmean left lung of $16.9 \mathrm{~Gy}$ ), thus we could reduce the dose to the left lung to around $4.5 \mathrm{~Gy}$ even in this special case. The treatment was well tolerated. Afterwards a close follow-up care quarterly was performed in the first 24 months. In this periode the patient developed neither radiation pneumonitis nor lung fibrosis.

Additionally, the DIBH technique reduced the Dmean of the heart compared to NB. The average coverage of Dmean heart was $1.17 \mathrm{~Gy}$ (Range $0.12 \mathrm{~Gy}-3.19 \mathrm{~Gy}$ ) in the DIBH cohort. $75 \%$ of all patients were able to reach a Dmean under $1.55 \mathrm{~Gy}$. The NB plans had a Dmean heart of 2.31 Gy (Range 0.71-4.21 Gy), which means that the DIBH-technique could halve the Dmean heart according to literature $[16,39,54-58]$. The maximum heart distance (maximal thickness of heart irradiated) was mostly less than $1 \mathrm{~cm}$ (Range $0.8-1.2 \mathrm{~cm}$ ) during irradiation of our DIBH breast cancer cases, mostly less than $2 \mathrm{~cm}$ (Range $1.1-3.2 \mathrm{~cm}$ ) in the NB plans.

In summary the Dmean heart could be reduced by $45.4 \%$ compared with the NB-technique and the Dmean left lung by $22.5 \%$ ( Fig. 5).

\section{Technical problems}

In our series this innovative technique had to deal with different problems concerning breathing conditions (e.g. abdominal breathing, nervous breathing, respiratory problems), the cogni- tive situation of the patient (e.g. memory performance, memory loss, dementia and many more) and the barrier of language. Even the marker block sometimes caused trouble (e.g. lack of signal, block lies in the treatment field). In daily routine this technique was associated with a higher amount of work, the assumption of additional responsibility for the special working group, a high expenditure of time and probably additional costs. We determined that the additional time we needed was up to 40 minutes according to our time schedule (Range 32-52 min) when performing the virtual simulation. Apart from this it needed up to 10 extra-minutes in daily routine per patient for treatment delivery due to the breathing commands and the complex set up. Although this technique was time consuming, needed patient cooperation and technical expertise it clearly reduced the doses to the heart and left lung. Similar findings have been reported in literature [20, 42, 47].

\section{Follow up}

Follow-up was carried out until June 2019, at that time all patients were still alive. Median follow-up for all patients was 52 months (range 7-73 m). No relevant differences were found between both treatment groups. Side effects were classified according to the Radiation Therapy Oncology Group/The European Organisation for Research [37], there was a slight difference between both arms, the NB arm had less RTOG 2 skin reactions (NB: $\mathrm{DIBH}=19.4 \%: 29 \%$ ). Apart from this the amount of the side effects was comparable (RTOG 1: $70.8 \%$ vs. $64 \%$, RTOG $36.6 \%$ vs. $5.6 \%$, no reaction $3.2 \%$ vs. $1.4 \%$ ). Late toxicity rates according to the Common Terminology Criteria for Adverse Events Version 4.03 (including teleangiectasia, skin and subcutaneous tissue disorders, skin atrophia, skin hyperpigmentation, pruritus, skin induration, skin ulceration) were distributed similarly, however we noted a higher teleangiectasia rate in patients who received a systemic therapy with chemotherapy and trastuzumab. Additionally there were 4 times more CTCAE 1 events in the NB-arm (51.6\% vs. $12.67 \%)$. One reason for this could be that the hypofractionation regimen was used in $82 \%$ of the NB-patients. Grade 3-5 late side effects did not occur in both arms. A detailed comparison of the group-specific toxicity results is depicted in $>$ Table 1, > Fig. 6. 

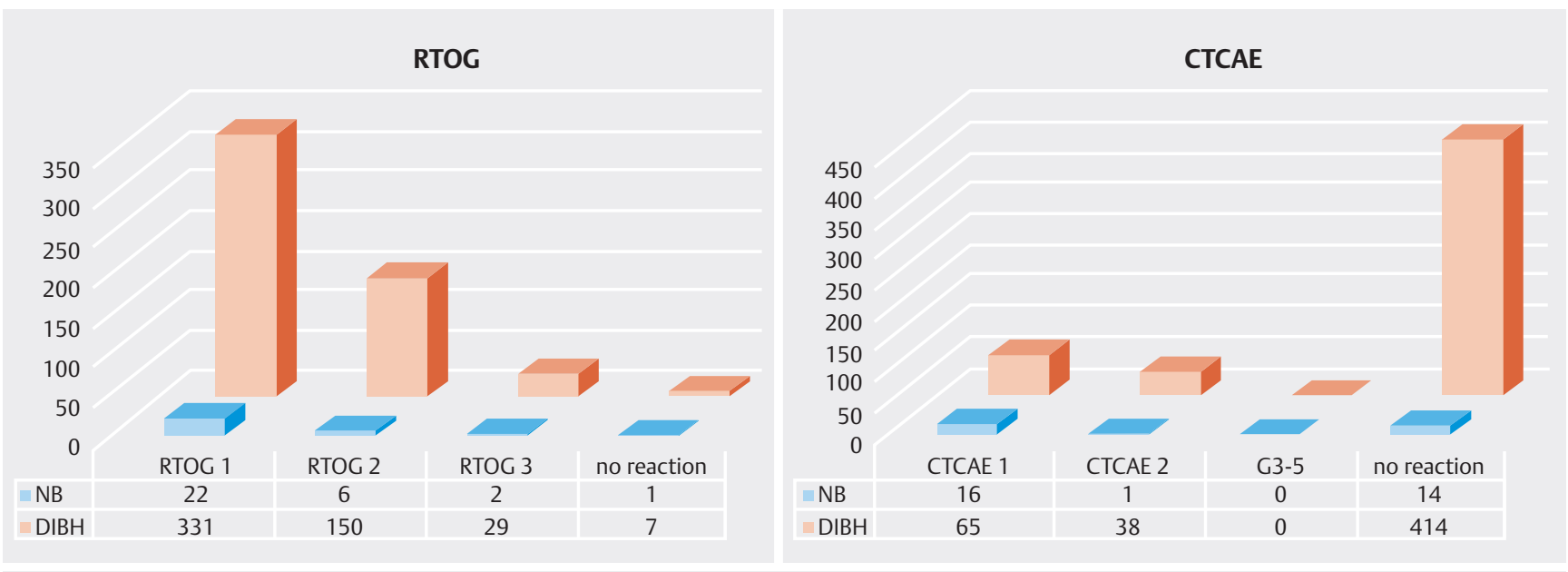

- Fig. 6 Comparison DIBH and NB patients: acute side effects RTOG-classification, late side effects CTCAE-criteria.

\section{Discussion}

Cardiac toxicity after breast cancer [16, 34, 48, 52], lung cancer $[53,54]$ and mediastinal lymphoma $[49,50,59]$ is the most reported radiation-induced complication [51]. The common clinical complications are asymptomatic pericarditis, congestive heart failure, and heart infarction. Even though clinicians have to give particular attention to these complications, anthracycline treatment [60-62]) is an additional major risk factor for additional cardiotoxicity during radiotherapy with a synergistic effect. However, the use of anthracycline, other cardiotoxic chemotherapies, immunotherapy [63-65] and targeted therapies [66, 67] should only be used with great caution and only after carrying out a careful treatment plan and optimization [1, 30, 40].

There is a wealth of evidence from retrospective and planning studies demonstrating reduction in dose to the heart and coronary arteries with DIBH treatment of left-sided breast cancers $[10,14,15]$. Additionally the total dose delivered to the planning target volume (PTV), the dose per fraction and the irradiated volume are correlated to the risk of cardiotoxicity. Remarkably, the volume of the heart receiving $35 \mathrm{~Gy}$ must be less than $30 \%$ and dose per fraction should not exceed 2 Gy when dose of prescription exceeds $30 \mathrm{~Gy}$ as previous studies confirmed [30]. On the one hand Darby et al. [16] showed a dose/effect-relationship with an increase in the relative risk of acute major coronary events of $7.4 \%$ per Gy (95\% confidence interval, Cl: 2.9-14.5; $\mathrm{p}<0.001$ ) mean heart dose within 20 years, on the other hand Van den Bogaard et al. [58] found a relative increase in the cumulative incidence of acute coronary events (ACE) of $16.5 \%$ per Gray(hazard ratio, HR: 1.165 ; $95 \%$ Cl for HR: 1.006 to 1.350 ; $p=0.042$ ) of mean heart dose within 9 years of RT.

Modern irradiation techniques seem to be associated with a limited risk of heart complication. Taylor et al. [21, 56, 57] comparatively analyzed mean heart doses from left tangential RT to cardiac structures over several decades, and described reductions in mean heart dose from $13.3 \mathrm{~Gy}$ in the $1970 \mathrm{~s}$, to $4.7 \mathrm{~Gy}$ in the 1990 s, and 2.3 Gy in 2006 due to major advances in RT tech- niques. Deep inspiration breath hold (DIBH) reduces heart and left anterior descending artery (LAD) dose during left-sided breast radiation therapy (RT); however there is limited information about which patients derive the most benefit from DIBH. Our study results are similar to published retrospective and planning studies demonstrating that DIBH lowered Dmean Heart and LAD doses $[2,4,8,10,14,15,41]$. Based on available findings, the DEGRO breast cancer expert panel recommends a Dmean heart dose $<2.5$ Gy [55]. Additionally, the study of Yeung et al. [39] showed that all patients receiving WBRT (whole breast irradiation) with DIBH met a Dmean heart <4 Gy and had less heart problems. Remarkably, in our series all patients with DIBH could reach a Dmean heart $<1.2$ Gy and almost all patients with NB a Dmean heart $<2.3$ Gy which means that our data could comply the DEGRO-recommendations.

However, proton therapy is another breast cancer radiation modality used to spare heart radiation exposure, taking advantage of the dosimetric properties of protons to reduce cardiac doses. Recent series have shown remarkably low cardiac doses with proton therapy [43]. Comparisons of protons at free-breathing versus photons with DIBH have shown that both techniques yield remarkably low heart doses, although proton plans appear to deliver lower mean heart dose and lower dose to the $\operatorname{LAD}[44,45]$. This may be answered by the PCORI RADCOMP trial [46]. This trial aims to enroll 1716 patients receiving radiotherapy to the breast or chestwall in conjunction with the internal mammary nodes and randomizes them to radiotherapy with either protons or photons. The primary endpoints of this trial are cardiac events and cancer control events.

Nevertheless limits of our analysis are the retrospective design, the lack of randomization and follow up in terms of recurrence probability. It is possible that the small sample sizes used in this retrospective analysis did not achieve the statistical power to show a significant effect. Further prospective studies with larger sample sizes are required to determine if there is a statistical difference between NB and DIBH and to determine threshold doses to cardiac structures in breast radiotherapy. 


\section{Conclusion}

To sum up the Deep inspiration breath-hold (DIBH) technique with Surface Guided Radiation Therapy (SGRT) is a rather simple method with a high acceptance by patients who can actively participate in the whole treatment process. With appropriate patient selection and adequate training the treatment delivery is acceptable and feasible. DIBH with SGRT should be considered for all patients receiving $\mathrm{RT}$ for left-sided breast cancer. In particular, patients with breast/chest wall RT plus nodal RT seem to benefit most. In our cohort the reproducibility and stability of the combination of DIBH with SGRT was evident.

\section{Conflict of Interest}

The authors declare that they have no conflict of interest.

\section{References}

[1] Corradini S, Ballhausen $\mathrm{H}$, Weingandt $\mathrm{H}$ et al. Left-sided breast cancer and risks of secondary lung cancer and ischemic heart disease. Strahlenther Onkol 2018; 194: 196-205

[2] Conway JL, Conroy L, Harper L et al. Deep inspiration breath-hold produces a clinically meaningful reduction in ipsilateral lung dose during locoregional radiation therapy for some women with right-sided breast cancer. Pract Radiat Oncol 2017; 7: 147-153

[3] Darapu A, Balakrishnan R, Sebastian P et al. Is the Deep Inspiration Breath-Hold Technique Superior to the Free Breathing Technique in Cardiac and Lung Sparing while treating both Left-Sided Post-Mastectomy Chest Wall and Supraclavicular Regions. Case rep Oncol 2017; 10: 37-51

[4] Lawler G, Leech M. Dose Sparing Potential of Deep Inspiration Breathhold Technique for Left Breast Cancer Radiotherapy Organs-at-risk. Anticancer Res 2017; 37: 883-890

[5] Jensen CA, Skottner N, Frengen J et al. Development of a deep inspiration breath-hold system for radiotherapy utilizing a laser distance measurer. J Appl Clin Med Phys 2017; 18: 260-264

[6] Koivumäki T, Tujunen J, Virén T et al. Geometrical uncertainty of heart position in deep-inspiration breath hold radiotherapy of left-sided breast cancer patients. Acta Oncol 2017; 56: 879-883

[7] Rice L, Goldsmith C, Grenne MMI et al. An effective deep-inspiration breath-hold radiotherapy technique for left-breast cancer: impact of post-mastectomy treatment, nodal coverage, and dose schedule on organs at risk. Breast Cancer 2017; 9: 437-446

[8] Sakka M, Kunzelmann L, Metzger M et al. Cardiac dose-sparing effects of deep-inspiration breath-hold in left breast irradiation. Strahlenther Onkol 2017; 193: 800-811

[9] Hepp R, Ammerpohl M, Morgenstern C et al. Deep inspiration breathhold (DIBH) radiotherapy in left-sided breast cancer. Strahlenther Onkol 2015; 191: 710-716

[10] Latty D, Stuart KE, Wang W et al. Review of deep inspiration breath-hold techniques for the treatment of breast cancer. J Med Radiat Sci 2015; 62: 74-81

[11] Rice L, Harris S, Green MML et al. Deep inspiration breath-hold (DIBH) technique applied in right breast radiotherapy to minimize liver radiation. BJR case reports 2015; 1: 20150038

[12] Whelan TJ, Olivotto IA, Parulekar WR et al. Regional nodal irradiation in early-stage breast cancer. N Engl J Med 2015; 373: 307-316
[13] Thorsen LB], Berg M, Brodersen $\mathrm{H}$ ] et al. Improved survival with internal mammary node irradiation: a prospective study on 3072 breast cancer patients. Radiother Oncol 2014; 111: 67-68

[14] Bruzzaniti V, Abate A, Pinnarò P et al. Dosimetric and clinical advantages of deep inspiration breath-Hold (DIBH) during radiotherapy of breast cancer. J Exp Clin Cancer Res 2013; 32: 88

[15] Nissen HD, Appelt AL. Improved heart, lung and target dose with deep inspiration breath hold in a large clinical series of breast cancer patients. Radiother Oncol 2013; 106: 28-32

[16] Darby SC, Ewertz M, McGale P et al. Risk of ischemic heart disease in women after radiotherapy for breast cancer. N Engl J Med 2013; 368: 987-998

[17] Haviland JS, Owen JR, Dewar JA et al. The UK Standardisation of Breast, Radiotherapy (START) trials of radiotherapy hypofractionation for treatment of early breast cancer: 10-yearfollow-up results of two randomised controlled trials. Lancet Oncol 2013; 14: 1086-1094

[18] Bouillon K, Haddy N, Delaloge S et al. Long-term cardiovascular mortality after radiotherapy for breast cancer. J Am Coll Cardiol 2011; 57: 445452

[19] Whelan TJ, Pignol JP, Levine MN et al. Long-term results of hypofractionated radiation therapy for breast cancer. N Engl J Med 2010; 362: 513-520

[20] Stranzl H, Zurl B. Postoperative irradiation of left-sided breast cancer patients and cardiac toxicity. Dose deep inspiration breath-hold (DIBH) technique protect the heart? Strahlenth Onkol 2008; 184: 354-358

[21] Taylor CW, Nisbet A, McGale P et al. Cardiac exposures in breast cancer radiotherapy 1950s-1990s. IJRBP 2007; 69: 1484-1495

[22] Correa CR, Litt HI, Hwang WT et al. Coronary artery findings after leftsided compared with right-sided radiation treatment for early stage breast cancer. JCO 2007; 25: 3031-3037

[23] Clarke M, Collins R, Darby S et al. Early Breast cancer Trialists'Collabortaive Group (EBCTCG): Effects of radiotherapy and of differences in the extent of surgery for early breast cancer on local recurrence and 15-year survival: an overview of the randomized trials. Lancet 2005; 366: 20872106

[24] Rutqvist LE, Johansson $\mathrm{H}$. Mortality by laterality of the primary tumour among 55000 breast cancer patients from the Swedish Cancer Registry. Br J Cancer 1990; 61: 866-868

[25] Schratter-Sehn AU, Schurawitzki H, Zach M et al. High-resolution computed tomography of the lungs in irradiated breast cancer patients. RadiotherOncol 1993; 27: 198-202

[26] https://www.varian.com/de/products/radiotherapy/real-time-trackingmotion-management/real-time-position-management

[27] C-RAD. Catalyst HD. Im Internet: https://c-rad.se/product/catalyst-hd. Stand: 19.12.2019

[28] http://embed.widencdn.net/pdf/view/varian/02uaz1vhfj/PerfectPitch6 DOF_ProductBrief_10264B_1013.pdf?u=wefire

[29] START Trialists' Group et al. The UK Standardisation of Breast Radiotherapy (START) Trial B of radiotherapy hypofractionation for treatment of early breast cancer: a randomised trial. Lancet 2018; 371: 1098-1107

[30] Doyen J, Giraud P, Belkacemi Y. Normal tissue tolerance to external beam radiation therapy: cardiac structures. Cancer Radiother 2010; 14 319-326

[31] Thill M, Liedtke C, Müller V et al. AGO Recommendations for the Diagnosis and Treatment of Patients with Advanced and Metastatic Breast Cancer: Update 2018. Breast Care (Basel) 2018; 13: 209-215. doi:10.1159/000489331

[32] AWMF. S3-Leitlinie Früherkennung, Diagnostik, Therapie und Nachsorge des Mammakarzinoms (Version 4.1, 20189). Im Internet: https://www. awmf.org/leitlinien/detail/II/032-045OL.html. Stand: 19.12.2019 
[33] White J, Tai A, Arthur D. Breast Cancer Atlas for Radiation Therapy Planning: Consensus Definitions RTOG (Radiation Therapy Oncology Group). www.rtog.org/CoreLab/ContouringAtlases

[34] Darby S, McGael P, Correa C et al. Effect of radiotherapy after breastconserving surgery on 10-year recurrence and 15-year breast cancer death: meta-analysis of individual patient data for 10801 women in 17 randomised trials. Lancet 2011; 378: 1707-1716

[35] Bentzen SM, Agrawal RK, Aird EGA et al. The UK Standardisation of Breast Radiotherapy (START) trial B of radiotherapy hypofractionation for treatment of early breast cancer: a randomised trial. Lancet 2008; 371: 1098-1107

[36] Bentzen SM, Agrawal RK, Aird EGA et al. The UK Standardisation of Breast Radiotherapy (START) trial A of radiotherapy hypofractionation for treatment of early breast cancer: a randomised trial. Lancet Oncol 2008; 9: 331-341

[37] Lopez E, Núnez MI, Guerrero MR et al. Breast Cancer Acute Radiotherapy Morbidity Evaluated by Different Scoring Systems. Breast Cancer Res Treat 2002; 73: 127-134

[38] Common Terminology Criteria for Adverse Events (CTCAE) Version 4.0. Published: May 28, 2009 (v4.03: June 14, 2010). Im Internet: https:// www.eortc.be/services/doc/ctc/CTCAE_4.03_2010-06-14_QuickRefer ence_5x7.pdf. Stand: 19.12.2019

[39] Yeung R, Conroy L, Long K et al. Cardiac dose reduction with deep inspiration breath hold for left-sided breast cancer radiotherapy patients with and without regional nodal irradiation. Radiol Oncol 2015; 10: 200. doi:10.1186/s13014-015-0511-8

[40] Mamounas EP, Bandos H, White JR et al. NRG Oncology/NSABP B-51/ RTOG 1304: Phase III trial to determine if chest wall and regional nodal radiotherapy (CWRNRT) post mastectomy (Mx) or the addition of RNRT to whole breast RT post breast-conserving surgery (BCS) reduces invasive breast cancer recurrence-free interval (IBCR-FI) in patients (pts) with pathologically positive axillary (PPAx) nodes who are ypN0 after neoadjuvant chemotherapy (NC). JCO.2019.37.15_suppl.TPS600

[41] Hayden AJ, Rains M, Tiver K. Deep inspiration breath hold technique reduces heart dose from radiotherapy for left-sided breast cancer with deep breath-holding. J Med Imaging Radiat Oncol 2012; 56: 464-472

[42] Bergom C, Currey A, Desai N et al. Deep Inspiration Breath Hold: Techniques and Advantages for Cardiac Sparing During Breast Cancer Irradiation. Front Oncol 2018; 8: 87. doi:10.3389/fonc.2018.00087

[43] Bradley JA, Dagan R, Ho MW et al. Initial report of a prospective dosimetric and clinical feasibility trial demonstrates the potential of protons to increase the therapeutic ratio in breast cancer compared with photons. Int J Radiat Oncol Biol Phys 2016; 95: 411-421

[44] Lin LL, Vennarini S, Dimofte A et al. Proton beam versus photon beam dose to the heart and left anterior descending artery for left-sided breast cancer. Acta Oncol 2015; 54: 1032-1039

[45] Patel SA, Lu HM, Nyamwanda JA et al. Postmastectomy radiation therapy technique and cardiopulmonary sparing: a dosimetric comparative analysis between photons and protons with free breathing versus deep inspiration breath hold. Pract Radiat Oncol 2017; 7: e377-e384

[46] MacDonald SM. Proton therapy for breast cancer: getting to the heart of the matter. Int J Radiat Oncol Biol Phys 2016; 95: 46-48

[47] Tanguturi SK, Lyatskaya y, Chen Y et al. Prospective assessment of deep inspiration breath-hold using 3-dimensional surface tracking for irradiation of left-sided breast cancer. Pract Radiat Oncol 2015; 5: 358-365

[48] Henson KE, McGale P, Taylor C et al. Radiation-related mortality from heart disease and lung cancer more than 20 years after radiotherapy for breast cancer. Br J Cancer 2013; 108: 179-182
[49] Mill WB, Baglan R], Kurichety P et al. Symptomatic radiation-induced pericarditis in Hodgkin's disease. Int J Radiat Oncol Biol Phys 1984; 10: 2061-2065

[50] Aleman BM, van den Belt-Dusebout AW, Klokman W] et al. Long-term cause-specific mortality of patients treated for Hodgkin's disease. J Clin Oncol 2003; 21: 3431-3439

[51] Schultz-Hector S, Trott KR. Radiation-induced cardiovascular diseases: is the epidemiologic evidence compatible with the radiobiologic data? Int J Radiat Oncol Biol Phys 2007; 67: 10-18

[52] Nitsche M, Pahl R, Huber K et al. Cardiac Toxicity after Radiotherapy for Breast Cancer: Myths and Facts. Breast Care (Basel) 2015; 10: 131-135

[53] Wang K, Eblan M], Deal AM et al. Cardiac Toxicity After Radiotherapy for Stage III Non-Small-Cell Lung Cancer: Pooled Analysis of Dose-Escalation Trials Delivering 70 to 90 Gy. J Clin Oncol 2017; 35: 1387-1394

[54] Ming X, Feng $Y$, Yang $C$ et al. Radiation-induced heart disease in lung cancer radiotherapy - A dosimetric update. Medicine (Baltimore) 2016; 95: e5051

[55] Piroth MD, Baumann R, Budach W et al. Heart toxicity from breast cancer radiotherapy. Strahlenther Onkol 2019; 195: 1-12

[56] Taylor CW, Nisbet A, McGale P et al. Cardiac doses from Swedish breast cancer radiotherapy since the 1950s. Radiother Oncol 2009; 90: 127135

[57] Taylor CW, Povall JM, McGale P et al. Cardiac dose from tangential breast cancer radiotherapy in the year 2006. Int J Radiat Oncol Biol Phys 2008; 72: 501-507

[58] Van den Bogaard VAB, Ta BDP, van der Schaaf A et al. Validation and modification of a prediction model for acute cardiac events in patients with breast cancer treated with radiotherapy based on three-dimensional dose distributions to cardiac substructures. J Clin Oncol 2017; 35: $1171-1178$

[59] Hahn E, Jiang H, $\mathrm{Ng} \mathrm{A}$ et al. Late Cardiac Toxicity After Mediastinal Radiation Therapy for Hodgkin Lymphoma: Contributions of Coronary Artery and Whole Heart Dose-Volume Variables to Risk Prediction. Int J Radiat Oncol Biol Phys 2017; 98: 1116-1123

[60] Veiga LHS, Curtis RE, Morton LM et al. Combined effect of radiotherapy and anthracyclines on risk of breast cancer among female childhood cancer survivors: A report from the Childhood Cancer Survivor Study (CCSS). Im Internet: https://ascopubs.org/doi/abs/10.1200/ JCO.2019.37.15_suppl.10053. Stand: 19.12.2019

[61] Feijen EAML, Font-Gonzalez A, van der Pal HJH et al. Risk and Temporal Changes of Heart Failure Among 5-Year Childhood Cancer Survivors: a DCOG-LATER Study. J Am Heart Assoc 2019; 8: e009122

[62] Berry G], Jorden M. Pathology of radiation and anthracycline cardiotoxicity. Pediatric Blood and Cancer 2005; 44: 630-637

[63] Upadhrasta S, Elias H, Patel K et al. Managing cardiotoxicity associated with immune checkpoint inhibitors. Chronic Dis Transl Med 2019; 5: 6-14

[64] Varricchi G, Galdiero MR, Marone G et al. Cardiotoxicity of immune checkpoint inhibitors. ESMO Open 2017; 2: e000247

[65] Tajiri K, leda M. Cardiac Complications in Immune Checkpoint Inhibition Therapy. Front Cardiovasc Med 2019; 6: 3

[66] Chang HM, Okwuosa TM, Scarabelli T et al. Cardiovascular Complications of Cancer Therapy: Best Practices in Diagnosis, Prevention, and Management. J Am Coll Cardiol 2017; 70: 2552-2565

[67] Perez EA. Cardiac Toxicity of ErbB2-Targeted Therapies: What Do We Know? Clinical Breast Cancer 2008; 8 (Suppl. 3): 114-120 